\title{
COVID-19 fear and performance of workers: A moderated mediation role of organizational support and mental wellbeing
}

\section{Erasmus Keli Swanzy ${ }^{a^{*}}$}

${ }^{a}$ University of Bologna, Italy

\begin{tabular}{l}
\hline C H R O N I C L E \\
\hline Article history: \\
Received: August 29, 2021 \\
Received in revised format: \\
October 92021 \\
Accepted: October 25, 2021 \\
Available online: \\
October 25, 2021 \\
\hline Keywords: \\
Employees' COVID-19 fear \\
Mental wellbeing \\
Anxiety \\
Depression \\
Organizational Support \\
Job Performance
\end{tabular}

\section{A B S T R A C T}

This study examines the influence of employees' COVID-19 fear on their performance through intervening mechanisms such as mental wellbeing and organizational support. A total of 446 workers from different banks in Ghana completed a self-reported survey. Findings from the analysis conducted with SPSS PROCESS macro (model 7) revealed that employees' COVID-19 fear did not have any direct negative influence on their performance but instead had an indirect effect on their performance via mental wellbeing (anxiety and depression). The findings further revealed that the indirect effect of employees' COVID-19 fear on their performance via mental wellbeing (anxiety and depression) was lower when organizational support was higher. Thus, this study recommends organizations to intensify employee-supportive measures to help reduce the adverse impact of employees' COVID-19 fear on their mental wellbeing and performance.

(C) 2022 by the authors; licensee Growing Science, Canada

\section{Introduction}

As the world gradually heals from the disastrous effect of the Coronavirus (COVID-19) pandemic, organizations are looking forward to recovering from their losses due to the lockdown and other COVID-19 measures. Hence, organizations would need employees to perform at their best to overcome these challenges. However, since we are not out of the woods yet and more importantly, the COVID-19 vaccines produced does not assure us 100\% protection against the COVID-19 infection (Kaplan \& Milstein, 2021), employees going to work each day may still be terrified of COVID-19 due to its high transmission and mortality rate. Not only are they afraid of the COVID-19, but employees may also be scared of getting infected and unwittingly transmitting it to their families and close friends (Labrague \& de Los Santos, 2020; Mo et al., 2020). Labrague and de Los Santos (2020) opined that when this fear is not managed correctly over time, COVID-19 fear might generate negative lasting consequences on employees' performance. In addition, COVID-19 fear may affect employees' decision-making abilities which may detrimentally affect their performance if it is left unmanaged (Ahorsu et al., 2020; Malik et al., 2021). Hence, further studies must research whether employees' COVID-19 fear significantly and negatively affects their performance, and more importantly, how it can be managed (Gunaydin, 2021; Sasaki et al., 2020). Yet, previous research specifically examining the influence of employees' COVID-19 fear on their performance is scarce. Among the few studies that have specifically investigated this topic, Erer (2020) and Gunaydin (2021) observed that employees' performance was negatively influenced by their COVID-19 fear. However, the relationship between employees' COVID-19 fear and performance may go beyond a straightforward relationship. Therefore, further investigations are needed to examine the underlying moderating and mediating mechanisms through which employees' COVID-19 fear affects their performance at the workplace. Doing this will enlighten

* Corresponding author.

E-mail address: e.k.swanzy@gmail.com (E. K. Swanzy)

(C) 2022 by the authors; licensee Growing Science, Canada doi: $10.5267 /$ j.msl.2021.10.005 
us on how employees' COVID-19 fear affects their performance at the workplace. More importantly, it will enlighten organizations on how to address employees' COVID-19 fear.

Furthermore, Li et al. (2020) and Malik et al. (2021) pointed out that the mental wellbeing of employees in this COVID-19 era has been negatively influenced severely by the emergence of the deadly disease. Also, a plethora of studies have confirmed that employees' COVID-19 fear was a predominant factor that negatively affected their mental wellbeing. For example, Sasaki et al. (2020) reported that employees' COVID-19 fear positively affected their degrees of anxiety and depression experienced. Similarly, Khattak et al. (2020) observed that employees' psychological distress such as anxiety and depression was positively influenced by their fear of COVID-19. Labrague and de Los Santos (2021) found that a high degree of employees' COVID19 fear resulted in increasing their psychological distress, such as anxiety and depression. Study findings by Chen and Eyoun (2021) also revealed that employees' COVID-19 fear contributed positively to their poor mental wellbeing. On the other hand, Labrague and de Los Santos (2021) hinted that the negative influence of employees' COVID-19 fear on their mental wellbeing might negatively affect several work outcomes, including their performance. This may be because higher degrees of anxiety and depression due to employees' COVID-19 fear may cause further adverse cognitive responses involving low employees' vitality in performing their assigned jobs leading to low performance at the workplace (Gunaydin, 2021; Smith \& Wesselbaum, 2020). Also, Agular-Quintana et al. (2021) explain that poor mental wellbeing such as high anxiety and depression experienced by employees diminishes their cognitive resources and negatively affects their cognitive abilities, leading to poor performance of employees. Thus, indicating that employees' mental wellbeing could be a potential mediator between their fear of COVID-19 and their performance. However, no studies until now have investigated such a relationship. Therefore, the current study seeks to fill this research gap by examining how employees' COVID-19 fear influences their performance through two negative dimensions of mental wellbeing (anxiety and depression).

Moreover, a review of studies by Giorgi et al. (2020) pointed out that workplace factors could mitigate or worsen the adverse influence of employees' COVID-19 fear on their mental wellbeing. Shanafelt, Ripp, and Trockel (2020) identified that employees' doubt that their organizations would support them if they contracted COVID-19 contributed significantly to the levels of anxiety and depression they experienced. Therefore, support organizations provide in this COVID-19 era may be crucial in mitigating the adverse influence of employees' COVID-19 fear on their mental wellbeing and eventually improving their performance at the workplace (Giorgo et al., 2020; Sasaki et al. 2020). Similarly, Allah (2021) observed that high perceived organizational support diminished the impact of COVID-19 on the challenges that employees experienced in this COVID pandemic era. Possibly, the support employees receive from the organization has been known to improve their mental wellbeing (Caesens et al., 2017; Swanzy, 2020; Wattoo, Zhao \& Xi, 2018), and as employees' mental wellbeing improves, the adverse effect of fear of COVID-19 on them diminishes (Maslakçı, Sürücü \& Sesen, 2021). Yet, there is a paucity of studies explicitly examining the crucial role of organizational support on this topic (Zhang et al., 2020). Therefore, this research is vital as it seeks to provide empirical evidence on the crucial role organizational support may play to moderate the adverse influence of employees' COVID-19 fear on their mental wellbeing and performance.

To sum it all, the study examines the influence of employees' COVID-19 fear on their performance as mediated by two negative dimensions of mental wellbeing (anxiety and depression). In addition, it seeks to examine the mitigating influence of organizational support on this association. More precisely, this study has three objectives. To investigate (i) whether employees' COVID-19 fear negatively affected their performance; (ii) whether anxiety and depression mediated the negative relationship between employees' COVID-19 fear and their performance; and (iii) whether organizational support moderated the indirect effect of employees' COVID-19 fear on their performance via anxiety and depression. The findings of this study will enlighten us in understanding the underlying moderated and mediated mechanisms through which employees' COVID19 fear affects their performance. Furthermore, this will offer insights to organizational management to implement more employee-supportive policies to buffer the deleterious influence of employees' COVID-19 fear.

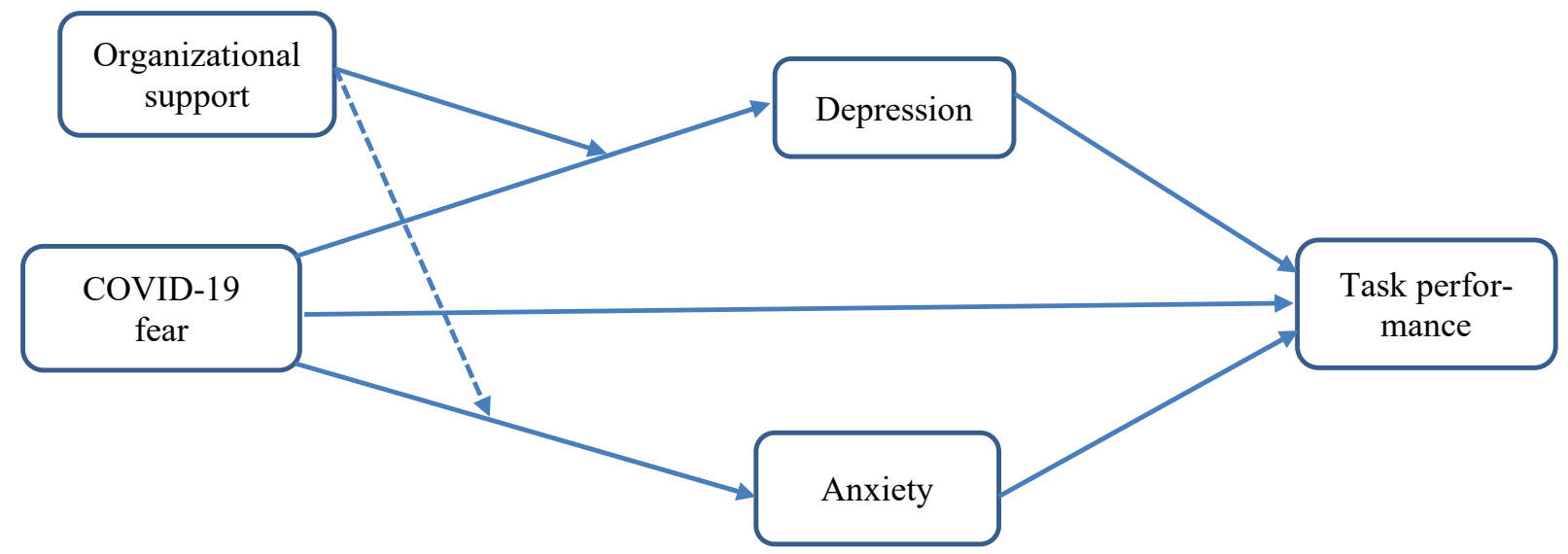

Fig. 1. Proposed Research Model 


\section{Methodology}

\subsection{Sample and Procedure}

This current research is a cross-sectional one. The sample of this research included bank workers in Accra, Ghana. Selfreported surveys were administered in person to several workers of different banks and branches, including Ghana Commercial Bank, Fidelity Bank, Prudential Bank, and Agricultural Rural Bank. In all, 476 workers answered to the survey. After, the data was then screened for missing data, and uncompleted questionnaires were deleted, resulting in a final sample size of 446. Of the 446 workers who responded to the survey, 215 were women, while the remaining 231 were men, representing $48.2 \%$ and $51.8 \%$. Participants' minimum and maximum ages were 24 and 65 years, respectively, with their mean age of 45.77 years $(\mathrm{SD}=11.02)$. Regarding their marital status, $278(62.34 \%)$ reported being married, while the remaining 168 $(37.66 \%)$ indicated not being married.

\subsection{Measures}

Fear of COVID-19: Employees' COVID-19 fear were measured using the Fear of COVID-19 Scale (FCV-19S) by Ahorsu et al. (2020). The total items of this scale are seven and five responses measured on a Likert scale ranging from " $1=$ strongly disagree to 5 = strongly agree". Samples of the items comprise: "I am most afraid of coronavirus-19", "It makes me uncomfortable to think about coronavirus-19", and "I am afraid of losing my life because of coronavirus-19".

Anxiety: Employees' anxiety was quantified using the Generalized Anxiety Disorder Scale (GAD-7) by Spitzer et al. (2006). The scale consisted of seven items and four responses measured on a Likert scale from " $0=$ Not at all to $3=$ Nearly every day". Samples of these scale items are: "Over the last two weeks, how often have you been bothered with the following problems?" "Troubling relaxing", "Feeling nervous anxious or on edge", and "Not being able to stop worrying or control worrying".

Depression: Depression of workers were measured utilizing the Patient Health Questionnaire (PHQ-9) by Kroenke et al. (2001). The scale comprises nine items and four responses measured on a Likert scale varying from " $0=$ Not at all to $3=$ Nearly every day". Samples of these scale items are: "Over the last two weeks, how often have you been bothered with the following problems?" "Little interest or pleasure in doing things", "Feeling down, depressed or hopeless", and "poor appetite or overeating".

Performance: Employees' performance was assessed using the Task Performance dimension of the Individual Work Performance Questionnaire (IWPQ) by Koopmans et al. (2014). The scale included other performance dimensions assessed over the last three months. However, only the dimension that measures task performance was used for this study. The scale consisted of seven items and five responses on a Likert scale from " $1=$ strongly disagree to $5=$ strongly agree". Samples of this scale items are: "For the last three months"- "I was able to perform my work well with minimal time", "I managed to plan my work so that it was done on time", and "I know how to set the right priorities".

Organizational Support: The shorter edition of Eisenberger et al. (1986) Perceived Organizational Support Scale (POS-8) was employed to measure the support workers received from their respective organizations. The scale comprises eight items and five responses on a Likert scale from " $1=$ strongly disagree to $5=$ strongly agree". Samples of these scale items are: "The organization shows very little concern for me", "Help is available from the organization when I have a problem", and "The organization really cares about my wellbeing".

\subsection{Data Analysis}

A preliminary analysis testing the fitness of our research model, convergent validity, and common-method variance was computed before testing the study's hypotheses. This was done using Confirmatory Factor Analysis and Exploratory Factor Analysis. Also, descriptive statistics were computed to investigate the average mean, standard deviation and intercorrelations among study variables. Finally, SPSS PROCESS Macro (Model 7), with 5000 bootstrap samples, was used to examine the study objectives.

\section{Results}

\subsection{Measurement Model}

To test for the fitness of our research model, two measurement models, one-factor model and a five-factor model, were tested with a Confirmatory Factor Analysis and results compared. The results displayed in table 1 showed that the five-factor model which is the main research model of the study revealed a more satisfactory model fit $\left(\chi^{2}(657)=1689.33, \chi^{2} / \mathrm{df}=2.57 ; \mathrm{CFI}=\right.$ 0.94; TLI $=0.93$; RMSEA $=0.053 ; \mathrm{SRMR}=0.045)$ as compare to one-factor model where all 38 items were loaded on a single latent variable $\left(\chi^{2}(682)=7042.64, \chi^{2} / \mathrm{df}=10.03 ; \mathrm{CFI}=0.49 ; \mathrm{TLI}=0.46 ; \mathrm{RMSEA}=0.153 ; \mathrm{SRMR}=0.145\right)$. 


\subsection{Common-Method Bias}

The assessment of common-method bias was done adopting Harman's single-factor approach (1976). All items of the study variables were extracted into a single factor in Exploratory Factor Analysis. The results indicated a common-method bias of $28.47 \%$. This result is far lower than the acknowledged threshold of 50\% (Cooper et al., 2020; Doty \& Astakhova, 2020), signifying that there is no need to worry about common-method bias in this study.

\subsection{Descriptive Statistics}

Means, standard deviations, Cronbach's alpha and correlations of the present study variables are all reported in Table 1. The findings showed significant associations in line with the proposed model for the study. In other words, employees' COVID19 fear positively correlated with depression and anxiety, while it was negatively associated with their performance. In addition, anxiety and depression were also negatively associated with performance, while organizational support positively correlated with performance.

Table 1

Means, Standard Deviation, Pearson Correlations and Cronbach's alphas

\begin{tabular}{|c|c|c|c|c|c|c|c|}
\hline Variables & Mean & SD & 1 & 2 & 3 & 4 & \\
\hline 1. Covid-19 Fear & 3.51 & .87 & $(0.89)$ & & & & \\
\hline 2. Depression & 2.46 & .69 & $.24 * *$ & $(0.83)$ & & & \\
\hline 3. Anxiety & 2.31 & .93 & $.18^{* *}$ & $.29 * *$ & $(0.87)$ & & \\
\hline 4. Task Performance & 3.77 & .81 & $-.20 * *$ & $-.45 * *$ & $-.41 * *$ & $(0.81)$ & \\
\hline 5. Organizational Support & 3.03 & .79 & -.03 & $-.25 * *$ & $-.27 * *$ & $.35 * *$ & $(0.79)$ \\
\hline
\end{tabular}

Note. $* * \mathrm{p}<0.01, \mathrm{~N}=446$

\subsection{Main Findings}

A moderated mediated regression analysis using SPSS PROCESS macro (model 7) by Andrews Hayes was used to run the analysis for this study. The results presented in figure 2 indicated that employees' COVID-19 fear did not directly affect their performance $(\beta=-.04 ; p>.05)$. However, regarding the influence of employees' COVID-19 fear on their depression and anxiety levels, the results indicated that employees' COVID-19 fear had a significant positive influence on their depression $(\beta=.40 ; p<.001)$ and anxiety levels $(\beta=.33 ; p<.001)$. Furthermore, the results revealed that depression and anxiety of employees had a negative influence on their performance $(\beta=-.51 ; p<.001$ and $\beta=-.37 ; p<.001$ respectively).

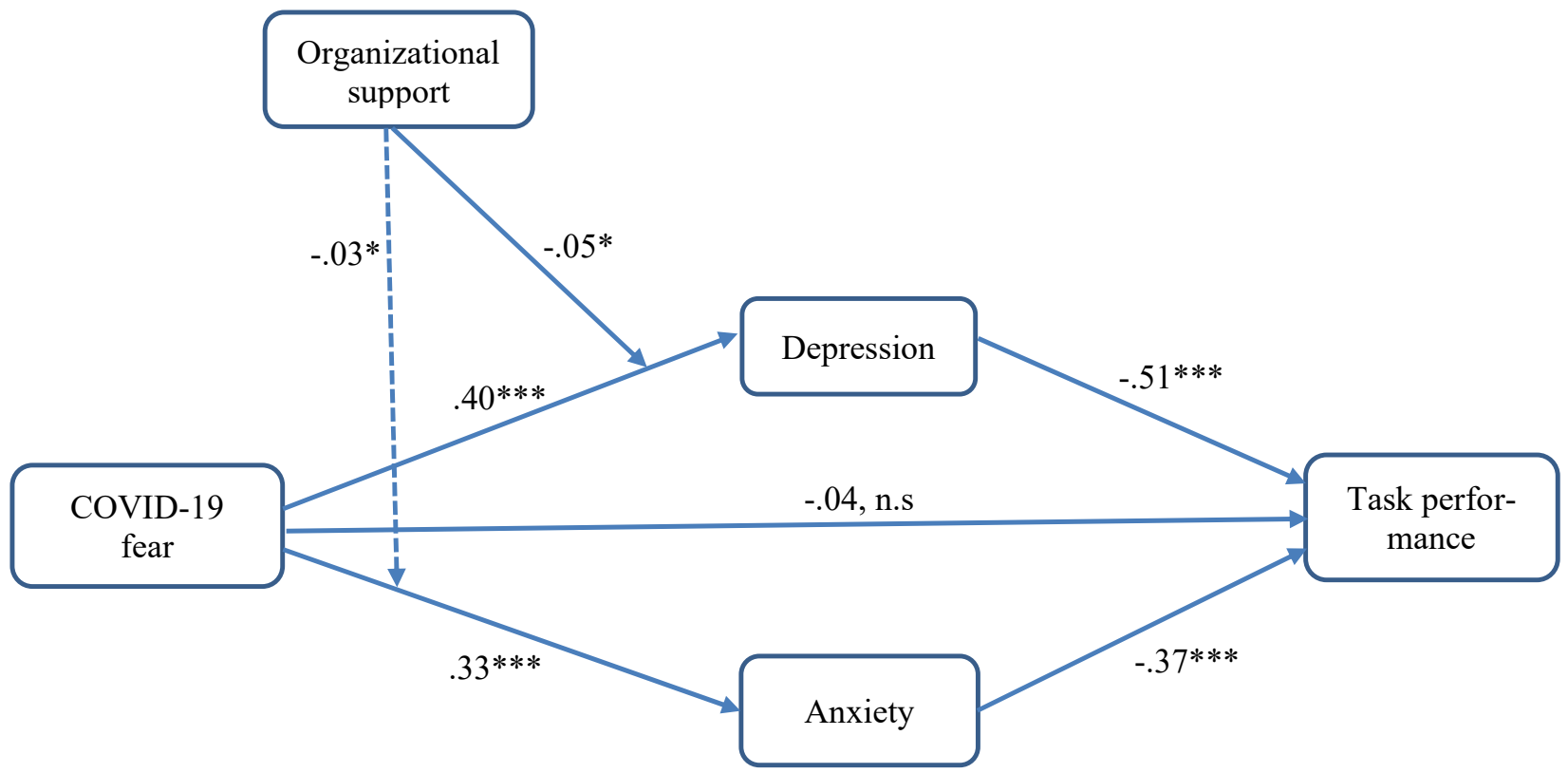

Fig 2. The model results show the effect of employees' COVID-19 fear on their performance as moderated by organizational support and mediated parallelly by anxiety and depression (standardized scores are reported) 


\subsubsection{The Moderating Role of Organizational Support}

Regarding the mitigating influence of organizational support on the negative impact of employees' COVID-19 fear on their levels of depression, the results from figure 2 indicated that organizational support moderated the positive impact of employees' COVID-19 fear on their levels of depression. Notably, the interaction term (computed as the product of the employees' COVID-19 fear and organizational support) appeared to have a significant negative effect on the depression of workers $(\beta=$ $-0.05, \mathrm{p}<0.05)$. Furthermore, the slope graph in figure 3 illustrated that the positive impact of employees' COVID-19 fear on their level of depression experienced was lower for employees who received high organizational support and was higher for employees who received low organizational support.

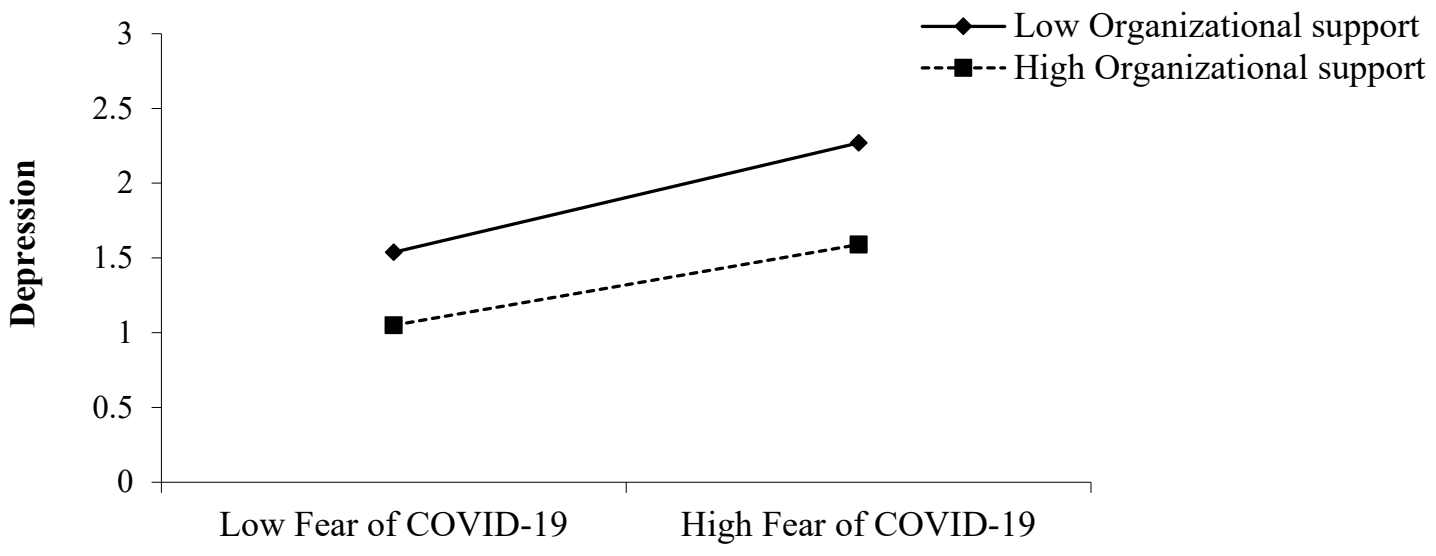

Fig. 3. Interaction effect of employees' COVID-19 fear and organizational support in predicting their depression levels

Likewise, the findings further indicated that organizational support moderated the positive impact of employees' COVID-19 fear on their anxiety levels. The interaction term (computed as the product of employees' COVID-19 fear and organizational support) appeared to have a significant negative impact on their levels of anxiety experienced $(\beta=-0.03, p<0.05)$. A graphical presentation of the outcome of this interaction is presented in Fig. 4. The slope graph in figure 4 illustrated that the positive impact of employees' COVID-19 fear on their anxiety was lower for employees who received high organizational support and was higher for employees with low organizational support.

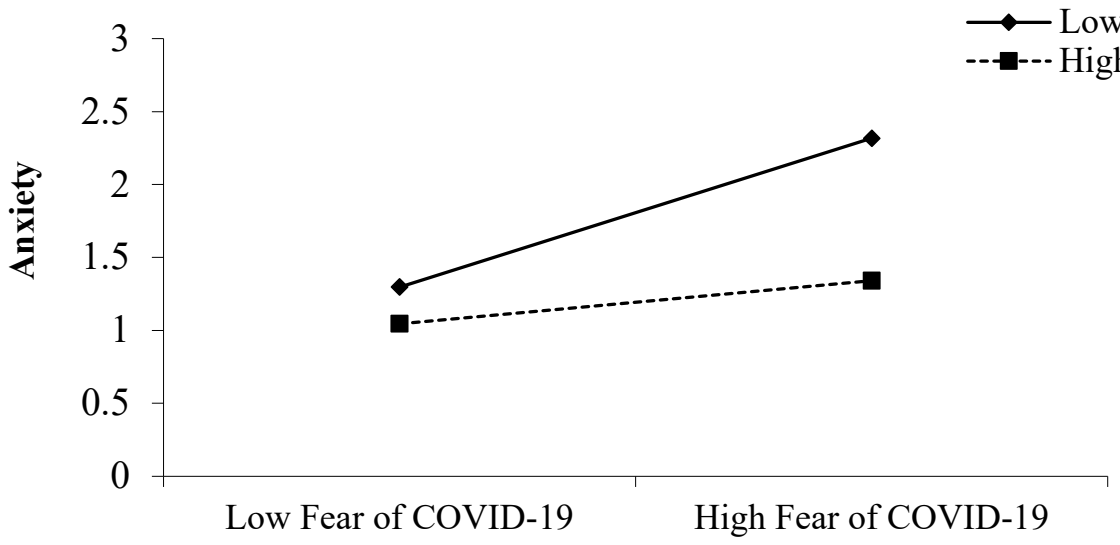

Fig. 4. Interaction between employees' COVID-19 fear and organizational support in predicting their anxiety levels

\subsubsection{The Mediating Role of Mental Wellbeing}

Table 3 shows the indirect effects of employees' COVID-19 fear on their performance through depression and anxiety. Specifically, regarding the mediating role of depression on the negative impact of employees' fear of COVID-19 and their performance, the findings indicated an indirect influence of employees' COVID-19 fear on their performance via depression ( $\beta$ $=-.061$; CI: $-.096,-.034)$. Similarly, the results indicated that the indirect effect of employees' COVID-19 fear on their performance through anxiety was significant $(\beta=-.043$; CI: -.067, -.015). 
Table 3

Indirect effects of employees' COVID-19 fear on their task performance via depression and anxiety

\begin{tabular}{lccc}
\hline & & \multicolumn{2}{c}{$95 \%$ CI } \\
\cline { 2 - 4 } Indirect effects & $\beta$ & LL & UL \\
\hline COVID-19 Fear $\rightarrow$ Depression $\rightarrow$ TP & -.061 & -.096 & -.034 \\
COVID-19 Fear $\rightarrow$ Anxiety $\rightarrow$ TP & -.043 & -.067 & -.015 \\
\hline
\end{tabular}

Note. $\mathrm{CI}=$ confidence interval; $\mathrm{LL} / \mathrm{UL}=$ lower/upper limit; $\mathrm{TP}=$ Task Performance

\section{Discussion}

Employees' COVID-19 fear did not directly impact employees' task performance, as reported by other studies (Erer, 2020; Gunaydin, 2021). A possible reason for this unexpected result may be due to where the research was conducted. This research was conducted in Ghana, which identifies itself among the countries that experienced low COVID-19 cases and deaths. Therefore, participants' COVID-19 fear may not be the same as participants from countries with high COVID-19 cases and deaths.

Rather, employees' COVID-19 fear contributed to increasing their anxiety and depression levels, which in turn affected their task performance. This supports suggestions made by Labrague and de Los Santos (2021), who opined that the adverse influence of employees' COVID-19 fear on their mental wellbeing might negatively affect several work outcomes, which may include their performance. Probably, employees' levels of anxiety and depression because of their COVID-19 fear may lead to further adverse mental responses such as avoidance behaviours affecting employees' vitality in performing their assigned jobs leading to low performance at the workplace (Malik et al., 2021). As Agular-Quintana (2021) explains, poor mental wellbeing such as high anxiety and depression experienced by employees diminishes their cognitive resources, negatively affecting their cognitive abilities and leading to poor performance of employees. Similar to our study findings, Malik et al. (2021) observed that employees' fear of COVI-19 significantly affected their anxiety and avoidance behaviours in the workplace, which may further alter their performances at work. Therefore, the study findings revealed that if organizations can focus on moderating the harmful influence of employees' COVID-19 fear on their mental wellbeing (anxiety and depression), it would play a critical role in diminishing the indirect negative effect of employees' COVID-19 fear on their performance.

Furthermore, the indirect effect of employees' COVID-19 fear on their task performance through depression and anxiety was weaker when employees received higher support from the organization. As observed from Figures 3 and 4, the positive influence of employees' COVID-19 fear on their anxiety and depression experienced was significantly lower when the support they received from their organizations was higher than when organizational support was lower. The findings of this study provide empirical support to studies that have advocated for higher organizational support in times like this (Allah, 2021; Shanafelt, Ripp, \& Trockel, 2020; Zhang et al., 2020). Shanafelt, Ripp, and Trockel (2020) contend that employees' scepticism that their organizations would support them if they contracted COVID-19 contributed substantially to the levels of anxiety they experienced. In addition, Sasaki et al. (2020) hinted that organizational support might play a crucial role in lessening the influence of employees' COVID-19 fear on their mental wellbeing, which may lead to better performance at the workplace. Our study findings support assertions made by Malik et al. (2021), who opined that lack of supportive organizations might worsen the indirect negative impact of employees' COVID-19 fear on their performance via mental wellbeing. It is clear now that when employees experience higher support from their organizations, it helps buffer the influence of their COVID-19 fear on their anxiety and depression experienced, which in turn reduced the negative effect on their task performance, leading to high performance at the workplace.

\subsection{Theoretical Implications}

This study answers the calls of previous studies (Sim, 2020) on the need for additional studies to investigate the influence of COVID-19 on employees' performance. The study outcomes demonstrated that employees' COVID-19 fear did not directly influence their performance but instead had an indirect influence on their performance through their anxiety and depression. This provides empirical support to previous research (e.g., Agular-Quintana, 2021; Labrague \& de Los Santos, 2021; Malik et al., 2021) that hinted at such a relationship and adds to the limited existing literature on the association between employees' COVID-19 fear and performance. Furthermore, there have been some suggestions by earlier studies (e.g., Allah, 2021; Shanafelt, Ripp, \& Trockel, 2020; Zhang et al., 2020) that the support employees may obtain from their organization may be critical in how employees' COVID-19 fear affected them. The study findings showed that organizational support reduced the influence of employees' COVID-19 fear on their mental wellbeing, leading to increased performance.

\subsection{Practical Implications}

The study findings offer suggestions that would be of great enlightenment to organizational management in this COVID-19 era. First, organizational management should initiate pragmatic employee-supportive policies that aim to improve employees' 
mental wellbeing if organizations wish to improve or maintain employees' performance at work. As the study findings indicated, the positive impact of employees' COVID-19 fear on their anxiety and depression was mitigated by organizational support, which in turn helped reduce the negative impact on their performance. Therefore, in this COVID-19 era, improving employees' wellbeing could be the ideal solution in mitigating the negative influence of employees' COVID-19 fear, and organizational support could be the ideal way of enhancing employees' wellbeing.

\subsection{Limitations and Directions for Further Studies}

The current study investigated the adverse effect of employees' COVID-19 fear on only their task performance, ignoring other performance components such as contextual, adaptive, and counterproductive work performance. Hence, this may not serve as a true representation of employees' performance. Therefore, further studies should endeavour to examine the influence of employees' COVID-19 fear on all the dimensions of job performance. Also, the cross-sectional research design utilized for this study may limit the claims of the causal inferences revealed by the current study's findings. Hence, further research should make an effort to include time-lagged research designs in order to produce stronger causal inferences among the study variables.

\section{Conclusion}

This study investigated whether employees' fear of COVID-19 affected their task performance as mediated by mental wellbeing and moderated by organizational support. The study findings revealed that improving employees' mental wellbeing was ideal for managing undesirable consequences of employees' COVID-19 fear on their performance. Furthermore, organizational support may be instrumental in improving employees' mental wellbeing by buffering the negative effect of their COVID-19 fear on their mental wellbeing. Therefore, a takeaway note for organizations will be that organizations should increase employee-supportive measures during this time of the COVID-19 pandemic to help reduce the adverse influence of employees' COVID-19 fear on their mental wellbeing and performance.

\section{References}

Aguiar-Quintana, T., Nguyen, H., Araujo-Cabrera, Y., \& Sanabria-Díaz, J. M. (2021). Do job insecurity, anxiety and depression caused by the COVID-19 pandemic influence hotel employees' self-rated task performance? The moderating role of employee resilience. International Journal of Hospitality Management, 94, 102868.

Ahorsu, D. K., Lin, C. Y., Imani, V., Saffari, M., Griffiths, M. D., \& Pakpour, A. H. (2020). The fear of COVID-19 scale: development and initial validation. International journal of mental health and addiction, 1-9.

Bakioğlu, F., Korkmaz, O., \& Ercan, H. (2020). Fear of COVID-19 and positivity: mediating role of intolerance of uncertainty, depression, anxiety, and stress. International journal of mental health and addiction, 1-14.

Caesens, G., Stinglhamber, F., Demoulin, S., \& De Wilde, M. (2017). Perceived organizational support and employees' wellbeing: The mediating role of organizational dehumanization. European Journal of Work and Organizational Psychology, 26(4), 527-540.

Chen, H., \& Eyoun, K. (2021). Do mindfulness and perceived organizational support work? Fear of COVID-19 on restaurant frontline employees' job insecurity and emotional exhaustion. International Journal of Hospitality Management, 94, 102850 .

Cooper, B., Eva, N., Fazlelahi, F. Z., Newman, A., Lee, A., \& Obschonka, M. (2020). Common method variance in vocational behavior research: Reviewing the state of the literature. Journal of Vocational Behavior, 103472.

Deniz Günaydin, H. (2021). Impacts of Personality on Job Performance Through COVID-19 Fear and Intention to Quit. Psychological Reports, 00332941211040433.

Doty, D. H., \& Astakhova, M. (2020). Common method variance in international business research: A commentary. In Research methods in international business (pp. 399-408). Palgrave Macmillan, Cham.

Eisenberger, R., Huntington, R., Hutchison, S., \& Sowa, D. (1986). Perceived organizational support. Journal of Applied psychology, 71(3), 500-507.

Erer, B. (2020). Impact of Covid-19 Fear on Employee Performance. Social Sciences, 10(4), 845-852.

Gab Allah, A. R. (2021). Challenges facing nurse managers during and beyond COVID-19 pandemic in relation to perceived organizational support. In Nursing Forum. 56: 539-549.

Giorgi, G., Lecca, L. I., Alessio, F., Finstad, G. L., Bondanini, G., Lulli, L. G., ... \& Mucci, N. (2020). COVID-19-related mental health effects in the workplace: a narrative review. International journal of environmental research and public health, 17(21), 7857.

Harman, H. H. (1976). Modern factor analysis. University of Chicago Press, Chicago, IL.

Kaplan, R. M., \& Milstein, A. (2021). Influence of a COVID-19 vaccine's effectiveness and safety profile on vaccination acceptance. Proceedings of the National Academy of Sciences, 118(10), 1-5.

Khattak, S. R., Saeed, I., Rehman, S. U., \& Fayaz, M. (2020). Impact of fear of COVID-19 pandemic on the mental health of nurses in Pakistan. Journal of Loss and Trauma, 1-15.

Koopmans, L., Bernaards, C. M., Hildebrandt, V. H., De Vet, H. C., \& Van der Beek, A. J. (2014). Construct validity of the individual work performance questionnaire. Journal of occupational and environmental medicine, 56(3), 331-337. 
Kroenke, K., Spitzer, R. L., \& Williams, J. B. (2001). The PHQ-9: validity of a brief depression severity measure. Journal of general internal medicine, 16(9), 606-613.

Labrague, L. J., \& de Los Santos, J. A. A. (2021). Fear of Covid-19, psychological distress, work satisfaction and turnover intention among frontline nurses. Journal of nursing management, 29(3), 395-403.

Li, Z., Ge, J., Yang, M., Feng, J., Qiao, M., Jiang, R., ... \& Yang, C. (2020). Vicarious traumatization in the general public, members, and non-members of medical teams aiding in COVID-19 control. Brain, behavior, and immunity, 88, $916-919$.

Malik, S., Ullah, I., Irfan, M., Ahorsu, D. K., Lin, C. Y., Pakpour, A. H., ... \& Minhas, R. (2021). Fear of COVID-19 and workplace phobia among Pakistani doctors: A survey study. BMC Public Health, 21(1), 1-9.

Maslakçı, A., Sürücü, L., \& Sesen, H. (2021). Fear of COVID-19 and work-quality of life among nurses: The mediating role of psychological wellbeing. Management Science Letters, 11(7), 1985-1990.

Mo, Y., Deng, L., Zhang, L., Lang, Q., Liao, C., Wang, N., ... \& Huang, H. (2020). Work stress among Chinese nurses to support Wuhan in fighting against COVID-19 epidemic. Journal of nursing management, 28(5), 1002-1009.

Sasaki, N., Kuroda, R., Tsuno, K., \& Kawakami, N. (2020). The deterioration of mental health among healthcare workers during the COVID-19 outbreak: A population-based cohort study of workers in Japan. Scandinavian journal of work, environment \& health, 46(6), 639-644.

Shanafelt, T., Ripp, J., \& Trockel, M. (2020). Understanding and addressing sources of anxiety among health care professionals during the COVID-19 pandemic. Jama, 323(21), 2133-2134.

Smith, M. D., \& Wesselbaum, D. (2020). COVID-19, food insecurity, and migration. The Journal of Nutrition, 150(11), 28552858.

Spitzer, R. L., Kroenke, K., Williams, J. B., \& Löwe, B. (2006). A brief measure for assessing generalized anxiety disorder: the GAD-7. Archives of internal medicine, 166(10), 1092-1097.

Swanzy, E. K. (2020). The Impact of Supervisor Support on Employees' Psychological Wellbeing: A Parallel Mediation Analysis of Work-To-Family Conflict and Job Satisfaction. International Business Research, 13(11), 41-53.

Wattoo, M. A., Zhao, S., \& Xi, M. (2018). Perceived organizational support and employee wellbeing: Testing the mediatory role of work-family facilitation and work-family conflict. Chinese Management Studies, 12(2), 469-484.

Zhang, S. X., Sun, S., Jahanshahi, A. A., Alvarez-Risco, A., Ibarra, V. G., Li, J., \& Patty-Tito, R. M. (2020). Developing and testing a measure of COVID-19 organizational support of healthcare workers-results from Peru, Ecuador, and Bolivia. Psychiatry research, 291, 113174.

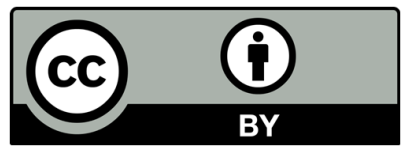

(C) 2022 by the authors; licensee Growing Science, Canada. This is an open access article distributed under the terms and conditions of the Creative Commons Attribution (CC-BY) license (http://creativecommons.org/licenses/by/4.0/). 